\title{
SEM and EDS Analysis Used in Evaluation of Chemical Pre-treatment Based on Nanotechno- $\log y$
}

Jaroslava Svobodova

Faculty of Production Technology and Management, J. E. Purkyne University in Usti nad Labem. Pasteurova 3334/7, 400 01 Usti nad Labem. Czech Republic. E-mail: svobodova@fvtm.ujep.cz

The most important aim of chemical pre-treatment is the removal of contaminants, corrosion products etc. from the material surface and obtaining such a basic material surface, which will provide sufficient corrosion resistance of the base material and suitable conditions for the adhesion of paints or other finish coatings to steel material. When selecting the method of chemical pre-treatment of the base material surface it should be considered whether the degree of preparation provides the required level of cleanliness of the surface and also the surface roughness and surface profile for the coating to be applied to the base material after chemical pre-treatment. The paper focuses on SEM and EDS analysis used in evaluation of chemical pre-treatment based on nanotechnology. These chemical pre-treatments are excluded on low carbon steel sheet. On the surface of low carbon steel sheet were excluded coatings of chemical pre-treatments (Fe phosphate, coating based on nanotechnology) and has been studied character of excluded layers on electron microscope.

Keywords: SEM, EDS analysis, chemical pre-treatment, phosphating, nanopassivation.

\section{Acknowledgments}

Author is grateful for the support of Internal student grant UJEP (IGA) No. 4820215000401.

\section{References}

[1] PHILIP, A., SCHWEITZER, P.E. (2005). Paint and Coatings: Aplications and Corrosion Reisistance, , pp. 672. Corrosion Technology - Svazek 23, Taylor\&Francis, ISBN 1574447025.

[2] CHOU, T. P., CHANDRASEKARAN, C., LIMMER, S., NGUYEN, C., CAO, G. Z. (2012). Organic-inorganic sol-gel rating for corrosion protection fo stainless steel, pp. 251 - 255. Journal of Materials Science, Letters 21, 2002, ISSN 0261-8028.

[3] MATERNE, T., BUYL, F., WITUCKI G. L. (2012). Organisilane Technology in Coating Application, pp. 16. Review and Perspectives, [online], [cit. 2012_07_25]. Dostupné z www: http://www.dowcorning.com/content/publishedlit/26-1402-01.pdf.

[4] VOEVODIN, A. A., SHTANSKY, D. V., LEVASHOV, E., A., MOORE, J. J. (2004). Nanostructured Thin Films and Nanodispersion Strengthened Coatings. Springer, Nato Science Series II: - Svazek 155, ISBN 1-40202221-2.

[5] KUŚMIERCZAK, S., SVOBODOVÁ, J. (2012). Microscopic Evaluation of Protective Coating by Coated Sheets after Corrosion Load, pp. 151-157. Manufacturing Technology, Journal for Science, Research and Production, Vol. 12, No. 13, ISSN 1213-2489.

[6] CAVAlEIRO, A., HOSSON, J. T. (2006). Nanostructured Coatings, pp. 648. Nanostructure Science and Technology, Springer, ISBN 0-387-25642-3.

[7] ADHIKARI, SAIKAT, K. A. UNOCIC, Y. ZHAI, G. S. FRANKEL, JOHN ZIMMERMAN \& FRISTAD (2011). Hexafluorozirconic acid based surface pretreatments: Characterization and performance assessment, pp. 1912-1924. Electrochimica Acta, roč. 56, č. 4, [online], [cit. 2013-07-01]. Dostupné z www: http://www.sciencedirect.com/science/article/pii/S0013468610009692

[8] KUŚMIERCZAK, S., MICHNA, Š. (2011). Analýza korozního poškození povrchu hliníkových materiáli dlouhodobým skladováním, pp. 32-36. Strojírenská technologie, Vol. 4, No. 4, ISSN: 1211-4162.

[9] WEISS, V., STŘIHAVKOVÁ, E. (2012). Influence of the homogenization annealing on microstructure and mechanical properties of AlZn5,5Mg2,5Cu1,5 alloy, pp. 297 - 302. Manufacturing Technology, Vol.12, No. 13, ISSN 1213 -2489.

[10] VALÁŠEK, P., MÜLLER, M. (2012). Influence of bonded abrasive particles size on wear of polymeric particle composites based on waste, pp. 268 - 272. Manufacturing Technology, roč. 12, č. 13. ISSN: 1213-2489.

[11] MÜLLER, M., NÁPRSTKOVÁ, N. (2010). Possibilities and limits of adhesive layer thickness optical evaluation, s. 45 - 49. Manufacturing Technology, roč. 10, č. 10, ISSN: 1213-2489. 
[12] MICHNA, Š., NÁPRSTKOVÁ, N. (2009). Vliv vnějších faktorů na korozni poškození hliníkových polotovarů, s. 17-21. Strojírenská technologie, roč. 14, č. 2, ISSN: 1211-4162.

[13] NDREATTA, F. a kol. (2011). Development and industrial scale-up of ZrO2 coatings and hybrid organicinorganic coatings used as pre-treatments before painting aluminium alloys, pp. 3 - 14. Progress in Organic Coatings, Vol. 72, Issues 1 - 2, [online], [cit. 2013-04-16]. Dostupné z www: http://www.sciencedirect.com/science/article/pii/S0300944011000294

\section{Paper number: M201486}

Copyright (C) 2014. Published by Manufacturing Technology. All rights reserved. 